\title{
El sistema nacional de salud del Perú. Entrevista al Dr. David Tejada de Rivero
}

\section{The national health system of Peru. Interview with David Tejada de Rivero MD PhD}

\author{
Wilson Ricardo Cañizares Fuentes ${ }^{1,2}$ \\ Universidad Católica de Santiago de Guayaquil. Guayaquil, Ecuador. \\ 2 Universidad de Guayaquil. Guayaquil, Ecuador.
}

\author{
Correspondencia: \\ Wilson Ricardo Cañizares Fuentes \\ wcanizar@hotmail.com
}

Recibido: 15 de febrero 2019

Aprobado: 7 de marzo 2019

Publicación en línea: 27 de marzo 2019

Conflictos de interés: El autor declara no tener conflictos de interés

Fuente de financiamiento:

Autofinanciado

Fuentes de financiamiento:

Autofinanciado

Citar como: Cañizares W. El Sistema Nacional de Salud en el Perú. Entrevista al Dr. David Tejada de Rivero. An Fac med. 2019;80(1):114-22.

DOI: https://doi.org/10.15381/anales. v80i1.15881

\section{An Fac med. 2019; 80(1):114-22. / DOI: https://doi.org/10.15381/anales.v80i1.15881}

\section{Resumen}

A propósito de mi tesis doctoral: "Evolución del sistema de salud de Perú, 2005-2014. Buenas prácticas de gestión", de la Facultad de Medicina, Universidad Nacional Mayor de San Marcos, del año 2017, realicé una entrevista a profundidad al Dr. David Tejada de Rivero en junio de 2015. En ocasión anterior ya le habia realizado una entrevista sobre la Atención Primaria de la Salud y su rol en la declaración de Alma Ata, disponible en YouTube. La tesis buscaba entender y explicar los avances y desafíos del sistema nacional de salud del Perú, las buenas prácticas y los factores que influyeron en su implementación. Para alcanzar ese propósito, en base a la metodologia de investigación cualitativa se consultaron las opiniones, discurso y reflexiones de altos directivos del Ministerio de Salud, expertos de salud pública, académicos y representantes de la sociedad civil peruana, entre los que estaba incluido el Dr. David Tejada. La entrevista fue realizada en su departamento en Miraflores, Lima, grabada y transcrita para realizar su posterior análisis. La entrevista fue previamente consultada y concertada con el entrevistado, así como todas las entrevistas que se hicieron a propósito del estudio. Por considerar de interés académico e histórico se consideró la importancia de publicar la entrevista completa del Dr. David Tejada de Rivero como homenaje y reconocimiento a sus importantes contribuciones a la salud publica peruana y mundial.

Palabras clave: Sistemas de Salud; Entrevista; Perú

\begin{abstract}
About my doctoral thesis: "Evolution of the health system of Peru, 2005-2014. Good management practices ", from the Facultad de Medicina, Universidad Nacional Mayor de San Marcos, in 2017, i conducted an in-depth interview with Dr. David Tejada de Rivero in june 2015. On the previous occasion I had already interviewed him Primary Health Care and its role in the Alma Ata declaration, available on YouTube. The thesis sought to understand and explain the advances and challenges of the national health system of Peru, good practices and the factors that influenced its implementation. To achieve this purpose, based on the qualitative research methodology, the opinions, discourse and reflections of senior executives of the Ministry of Health, public health experts, academics and representatives of Peruvian civil society were consulted, including Dr. David Tejada The interview was conducted in his apartment in Miraflores, Lima, recorded and transcribed for further analysis. The interview was previously consulted and agreed upon with the interviewee, as well as all the interviews that were made about the study. Considering the academic and historical interest, the importance of publishing the complete interview of Dr. David Tejada de Rivero as a tribute and recognition of his important contributions to Peruvian and world public health was considered.
\end{abstract}

Keywords: Health Systems; Interview; Peru 
Estamos haciendo la entrevista a propósito del estudio referente a la evolución en los últimos 10 años del sistema de salud del Perú. Usted ha sido Ministro de Salud, ex alto funcionario de la Organización Mundial de la Salud. Dr. Tejada, ¿puede hacer un breve resumen de su hoja de vida, identificando las principales funciones que usted ha realizado?

Yo le voy a mandar la hoja de vida por correo electrónico, es una sola página nada más, porque hoja de vida es distinta del currículo y ahora el currículo no necesita leerse, se mide en centímetros de altura de las páginas, porque entra todo. En cambio hoja de vida no es más de una página. Yo le voy a enviar mi hoja de vida para que vea mi experiencia de terreno y entre esa experiencia de terreno relacionada con el tema que usted está investigando.

Empecemos con lo que usted me decía previamente, que en este momento hay en vida 3 David Tejada...

Éramos cuatro David Tejada: mi padre David Tejada Mercado, yo David Tejada de Rivero, mi hijo David Tejada Pardo, que ha sido vice Ministro de Salud al comienzo del Gobierno de Fujimori y mi nieto que es diplomático de carrera y que ahora está de Secretario de la Embajada del Perú en Chile.

¿En qué año fue usted Ministro de Salud?

Fui dos veces, en el año 1985 a 1987 y después en el año 1989.

Perfecto, pero además usted fue funcionario de la Organización...

Fui Sub Director General de la Organización Mundial de la Salud.

Y ha ocupado muchísimos cargos importantes y recién veo que usted está formando parte de esta Organización No Gubernamental Consultores Internacionales de Salud y Desarrollo Social.

Soy fundador y Presidente de este grupo.

Y lo más importante, es que usted ha seguido de cerca y ha sido un actor protagónico de lo que ha acontecido en el Perú, en los últimos 20 a 30 años.

Yo estoy en el Perú; en este departamento, el primero de julio cumplo 19 años. Nunca he estado en un lugar tanto tiempo.

$Y$, ¿qué le parece estar nuevamente en Perú tanto tiempo, 19 años seguidos?

En mi patria, a pesar de que yo me considero arequipeño. Arequipa es como una república independiente. Ayer estábamos haciendo bromas con la Presidenta del Congreso, que es Ana María Solórzano y que ahora está metida en un pleito político grande... suspendió la reunión... todo eso... y la están atacando, le han presentado un voto de censura y ayer recién la conocí, nunca había estado cerca y le dije: "somos compatriotas". ¿Cómo? (me dijo ella). Claro-le dije- ¿o no es de Arequipa usted?. Por eso le digo compatriota, porque yo también soy de Arequipa. Porque nosotros somos de la "República Independiente de Arequipa, Amazónica, Antártica y con derecho a las Malvinas". Y todo es absolutamente cierto. Ya nadie discute, y la National Geographic tiene descrito y desarrollado, que el Amazonas nace en Arequipa, el rio Amazonas que es el río más largo del mundo.

\section{$A h$, pero ese rio nace en el Ecuador...}

El origen... Ios demás son afluentes. Pero el origen inicial del Amazonas es en Arequipa. Puede recurrir al National Geographic. Antártico, porque de acuerdo con las leyes internacionales cualquier país que tenga una costa entre dos meridianos y se prolonguen en el hemisferio sur, hacia el Polo Sur, tiene derecho a la Antártica, lo mismo ocurre para el otro. Y lo más importante, no lo sabía tampoco la Presidenta del Congreso, el derecho a las Malvinas. ¿Por qué?, es algo que pocos saben. Así como el primer presidente del Perú fue un argentino José de San Martin, el primer presidente de Argentina fue un arequipeño, Álvarez Thomas, y que fue el primer presidente argentino que reclamó las Malvinas; cuando él muere, deja en su testamento que todos los méritos que haya hecho en su vida, se los deja a su ciudad natal... Arequipa. Así que ino hay nada que hacer!.

Bien doctor, muy interesante. Nosotros queremos en este estudio, ver la evolución en los últimos diez años, ¿y porque diez años?, porque creemos que 10 años es un tiempo suficiente donde se podrían ver algunos cambios del sistema se salud de cada país. La época de estudio es del 2005 al 2014 y usted siempre ha sido uno de los actores clave, importante, opinando, participando en comisiones, haciendo recomendaciones. $Y$ el análisis del sistema de salud, a pesar de que hay varias clasificaciones y formas de ver el sistema de salud, nosotros hemos optado por ver tres componentes del sistema de salud: el primero es el tema de la rectoría, el tema de la gobernanza, de la regulación, de la legislación, de la conducción política del sistema. Entonces, queremos su opinión de cómo ha ido evolucionando eso, también el tema de la descentralización que es un tema que leí en los documentos que usted me envió.

Usted ve que soy crítico de eso (descentralización). Ha perjudicado. Todo el mundo dice que esto ha perjudicado y que hay dos responsables: Toledo y Alan García.

El segundo tema que queremos ver es el tema de las prestaciones, referente a la población que tenía acceso a atención de salud y prestaciones en el 2005. ¿Ahora es más amplia la cobertura?, ¿tiene acceso más cantidad de población?, ¿acceso a mejores cuidados, $o$ no?. Y el tercer componente es el financiamiento, ¿cómo ha evolucionado?. Estaba tanto en el 2005, ahora se habla que está alrededor del 2,8 o 3\% del PBI. El gasto de bolsillo, ahora se habla de alrededor del $40 \%$. Son temas que de alguna manera podrían permitir analizar la evolución del sistema de salud. En ese sentido, empecemos por la primera parte, en términos de la rectoría ¿cómo ve el Ministerio de Salud?.

Le voy a mandar un trabajo que tengo sobre la diferencia entre autoridad formal y liderazgo, y que tiene que ver con la función rectora del Ministerio de Salud. La función rectora del Ministerio de Salud tiene que tener una función de liderazgo nacional, en todos los sectores y a todos los niveles. No reducirse a los servicios de salud hospitalarios, centros de salud... no, no, no. Porque epidemiológicamente, cuando ustedes hacen un estudio preguntan ¿por qué? .... mejoró ¿por qué?... empeoró ¿por qué?. Lo que interesa es 
el por qué... sin explicación no hay nada, es simplemente decir cifras en el aire. Yo soy muy preocupado en el por qué. Entonces, la respuesta al tema es, rectoría significa el liderazgo que no se impone. Yo cuando fui ministro las dos veces, creé comités de coordinación intersectorial. Fue el inicio, no fue con la construcción que yo tengo ahora, y estaban representados los ministerios que tenían servicios de salud. Yo todavía confundía servicios de salud con salud... cosa que me rectifiqué después con mi experiencia mundial, de ser el Sub Director General Técnico de la OMS. Yo fui el Coordinador General de la Conferencia de Alma Ata, sobre Atención Primaria de Salud y tengo mucho escrito sobre Atención Primaria de Salud. Se ha distorsionado, se ha prostituido el término y se ha reducido a algo que es lo opuesto a la concepción que surgió de Alma Ata. Lo que diría es lo siguiente: La función rectora se ejerce por liderazgo y con la experiencia que yo tenía formé esos comités y teníamos sesiones cada dos semanas y en todas las sesiones llegábamos a acuerdos, pero notaba algo raro, y sobre todo en los representantes de los ministerios militares. En esa época no había un Ministerio de Defensa como ahora, había un Ministerio de Guerra, que era ejercito, marina y de aeronáutica; y la Policía en sus tres formas, Guardia Civil, Guardia Republicana y Policía de Investigaciones del Perú, eran separadas. Yo logré que vinieran representantes de todos estos, y claro, discutíamos y llegábamos a acuerdos, pero yo no veía, porque tenía mis contactos - porque uno no puede actuar en este mundo sin inteligencia, que no es la inteligencia mental, si no el conocimiento del enemigo, porque si uno no tiene el conocimiento del enemigo, está perdida la guerra, y eso es lo que se llama estrategia. Estrategia no es lo que yo voy a hacer, lo que yo voy a hacer es mi plan de guerra.

En base a lo que están pensando los otros...

Claro, y yo tengo que analizar si entro o no entro en guerra, cómo está el enemigo, en qué momento entro, y por dónde ataco y qué espero yo... es decir... la vida, los procesos sociales, son guerras, absolutamente guerras.
Pero son necesarios esos procesos sociales...

No es que son necesarios, no los inventamos. Los procesos sociales existen y nosotros somos partecita del proceso social. Ahí tengo también material que le puedo mandar sobre lo que son los procesos sociales. De lo que le he mandado, hay algo de lo que son los procesos sociales. Porque los procesos sociales son muy complejos.

$Y$ hay un tema que me gustó mucho, el tema que, en el fondo los sistemas de salud tienen que ver con decisiones políticas. En el fondo..

Todos los procesos sociales son materia de procesos políticos. Los procesos políticos son indispensables. Entendiendo la política en su verdadero sentido. La mejor definición de política que yo he encontrado -tengo un doctorado en ciencias políticas de la Universidad de Brasilia- ... no hablo porque sí tampoco. Si tuviera el doctorado solo y no la inquietud, es un título más, como quien dice que ya fui tres veces casado y tres veces divorciado.

Estábamos hablando del tema de la rectoría, y usted ¿cómo ve que ha evolucionado?

Como estaba inquieto para averiguar, dije: lo mejor para averiguar es con los ministros. Y a los ministros militares les gustaba tomar trago. Cuando nos encontrábamos en una embajada, yo no iba casi, pero comencé a ir para juntarme con ellos, los estimulaba a que tomaran trago, tomaran trago, y al final les decía: dime, dime ¿qué te dice tu representante?. Y no era un representante cualquiera, eran de nivel coronel, capitán de navío.

Se refiere a los representantes que mandaban para los temas de salud, que eran directores....

Eran los representantes de los ministros, que eran de confianza de ellos. Entonces: ¿qué te dicen?,-Bueno me dicen que todo está muy bien. Ya pues dime: ¿qué te dicen?, -Bueno, me dicen, mi general, he defendido la independencia de la institución para que no sea subordinada a un Ministerio de Salud... de tal manera que, participaban, aprobaban... iy no cumplían nada!.
Esa sería una de las razones porque es un sistema que se menciona fraccionado, cada quien...

Yo saque una conclusión desde esa época: todos somos partidarios de la coordinación mientras seamos coordinadores... y somos enemigos de la coordinación, mientras seamos coordinados... jes un complejo de inferioridad general!.

Pero hay un concepto interesante, que al final, el arte de la política no es el dominio de las mayorías sobre las minorías, sino la capacidad de llegar a consensos. ¿Cómo llegar a consensos a pesar de toda esta situación?

Ha leído lo que yo he escrito

\section{Si doctor, usted me lo facilitó}

Esa es la definición nada menos que de Sócrates, Platón y Aristóteles, escrita por Platón en el libro La Republica.

Que gracias a él tenemos esos escritos, porque los anteriores no escribían mucho, pero Platón sí sistematizó

Hay cosas interesantes ahí. Yo estudié mucho eso. ¿Por qué se llama academia?, ¿ested sabe?. Academia Nacional de Medicina, ¿ por qué se llama Academia?. Porque estos tres grandes filósofos, los más grandes que ha dado la historia de la humanidad, absolutamente insuperados hasta ahora, ellos marcaron todo lo que es la filosofía actual en el mundo y todos los valores y todas las cosas. Entonces, ellos eran estudiosos y profesores, y aplicaron la técnica que hoy día reclama Louis Ferrie y los autodenominados filósofos de la educación en Francia, como los más modernos... de 500 años antes de Jesús. Ellos no tenían donde reunirse con sus discípulos, y entonces un amigo de ellos que tenía una casa y un parque, un jardín, les facilitaba el jardín debajo de un árbol y entonces se sentaban alrededor... no estaban frente a una pizarra, ni tampoco con un power point, eso es más moderno, pero discutían, y ese era la mejor manera de enseñar, lo que están hoy día haciendo en China, en Shangai...

Conociendo la opinión de los otros, qué piensa la gente...

$Y$ discutiendo, discutir y discutir. $Y$ después le voy a decir la definición de la Escuela de Louis Ferrie, que retrata más 
o menos lo que era en esa época. Entonces, de esas discusiones salieron precisamente todas las grandes ideas que persisten hasta hoy en el mundo. ¿Por qué se llama Academia?, porque el dueño de la casa, del jardín y del árbol se llamaba Academus, por eso se llama Academia. Y academia significa eso... el método docente de Louis Ferrie...

Pero, regresando al Perú, allí este concepto de crear consensos en estos últimos diez años, hay un punto de inflexión que hemos escuchado y revisado, escuchando a quienes hemos ido haciendo las entrevistas, y lo sitúan alrededor del 2002, 2003, con el gobierno de Toledo, en el que se habla del gran Acuerdo Nacional, y al parecer ahí de alguna manera se llegó a poner algunos objetivos a largo plazo respecto de lo que quería el Perú sobre el tema del Sistema de Salud, y que allí marcaron algunas rutas que hasta ahora es el punto de inflexión y sirve como referente. Porque al final, como usted decía, la salud es un acto político y por lo tanto debe verse desde ese punto de vista.

Todo proceso social es político, todo

Coincidiría en que es importante analizar el Acuerdo Nacional...

El proyecto nacional es una cosa. En términos de ciencias políticas modernas, lo que se dice es, que no puede haber ninguna idea de futurismo, de planificación nacional, en una sociedad nacional, mientras no haya un proyecto nacional. $Y$ un proyecto nacional es a tiempo indefinido. El proyecto nacional permite recién, formar qué tipo de sociedad yo quiero llegar. Y para que sea un proyecto nacional, eso tiene que ser un consenso. Y un consenso significa que todos están de acuerdo, aunque tengan objeciones... esta parte no me gusta, esta parte estoy en contra... pero marchamos en la misma dirección... eso es consenso...

\section{Como un horizonte en común...}

Como un horizonte claro; y no solamente horizonte, es saber qué es lo que yo quiero al final. No es un horizonte desconocido. E incluso ese horizonte conocido, de la imagen que yo quiero para la proyección nacional, puede ir variando, porque con el tiempo y con la experiencia puedo ir modificando algunas cosas y puedo ir entendiendo otras y aprendiendo otras. Nadie es capaz de poder tener conocimientos totales de una materia para poder llegar así. Entonces otro de los elementos fundamentales en esto es la humildad. La humildad es de la época de Jesús, está en la Biblia, donde dice: "El valor más claro del desarrollo humano es la humildad".

¿Qué cree usted que se dio, en esos momentos, en esos años?. ¿Por qué se llegó a ese Acuerdo?

Entonces, el Acuerdo Nacional fue juntar a los partidos políticos, para ponerse de acuerdo ¿qué hacer en los próximos gobiernos?. Pero... nunca funcionó. $\mathrm{Ni}$ siquiera en el gobierno de Toledo. En elecciones, el que sale elegido ya... es igual... usted seguramente también tiene la experiencia que no es lo mismo.... ya no cambio de gobierno, cambio de ministro... Cuando hay el cambio de ministro, este ya no quiere saber nada de lo que hizo el anterior ministro, quiere hacer las cosas de nuevo, en otra dirección... no hay continuidad; y no hay continuidad porque no ha habido consenso. La garantía del consenso es que, si todos están de acuerdo, están obligados moralmente a seguir el acuerdo en la misma dirección, eso es lo que nos falta. Nos falta la humildad de decir: "Yo continuo lo que ha hecho el anterior, porque he participado".

Pero, puede ser que también el ministro no pueda continuar lo del anterior porque, si es un ministro de otro presidente, el otro presidente que ingresa, entra con otras ideas, también da ciertas directrices. En ese sentido, ¿es posible que el ministro de salud pueda posicionar algunas ideas en el presidente?

\section{¡Claro que sí!}

\section{$O$ tiene que convencer al ministro de economía, porque al final...}

Al final el poder está en quien tiene las finanzas, indudablemente; siempre ha sido así. En los ministerios, el ministro era cero frente a un director de administración. Yo lo he vivido cuando era asesor del ministro de salud, muy jovencito.
Hay una anécdota, que un ex ministro tuvo que encadenarse allí, en el ministerio de finanzas para que le puedan dar una partida presupuestal

Yo cometí una serie de errores, basándome en este estudio, cuando estaba en el poder porque el ministro que era Guillermo Garrido Lecca, me llamó de asesor sin conocerme, solamente por referencias. Igual que yo fui Sub Director General de la OMS, Ilamado por Halter Mayer sin conocerme y yo sin conocerlo. En esos momentos tratábamos de obtener información estadística, porque yo fui el primer director de la Oficina Nacional de Estadística de Salud, con financiamiento de Estados Unidos y con dos consultores americanos que vinieron a ayudarme. El presidente de la republica en esa época, pedía algo... ino hay!. O el ministro, siguiendo las indicaciones del presidente y el director de administración que era un señor Segovia, decía: jno hay!. Pero la esposa del presidente de la republica le pedía para alguna tontera... y isi hay!. Entonces, yo sabiendo eso.... ¿que hice?... me faltaba información... entonces yo me dije "acá la forma es esta"... Si esta información no me llega, le mandé decir oficialmente, no le va a girar la dirección.... y hable precisamente con Segovia y Segovia me dijo iperfecto!. Entonces, no le mandamos los fondos para pagar al personal, entonces al no tener fondos para pagar al personal tenían que mandarme la información... y funcionó "maravillosamente bien", me llegó la información, entonces la procesamos y la publicamos, pero después, como yo trataba de viajar y ver en el terreno ¿qué cosa era?, llegaba a un sitio.....pedía la información estadística, pero la información estadística... no... pero me han mandado la información estadística.... si pero.......y al final yo descubría que para poder recibir el dinero y para pagar al personal inventaban la información, iinventaban la información!. Y nosotros tontamente mezclábamos -yo con el apoyo de estos dos expertos norteamericanos - la información buena y legítima con la información falsa.... entonces, una tortilla con dos huevos podridos..... no se puede comer.... se malogra toda la tortilla. Hay que tener cuidado en la calidad y en la legitimidad de la información; no hay que 
creer la información, yo aprendí eso, que no hay que creer en la información...

Entonces, uno de los elementos claves de la rectoría deberían ser sistemas de información completa, transparente...

Es indispensable... iY muy bien controlados!. Porque esa fue mi experiencia... iterrible!. Yo cometí muchas cosas en mi vida, pero terribles, que si Dios es justo... yo me voy al infierno!... en serio.

Sobre el sistema de salud, ¿qué cosas, qué errores ha cometido?

Uyy....muchos errores. Felizmente tengo la capacidad de poder reconocer los errores, reconocer las fallas; y como le digo he cometido tantos errores, tan terribles que si hay justicia divina, me voy al infierno... pero no importa, me voy a encontrar con muchos amigos ahí....ja, ja, ja. Ahora, cuando hablamos de sistemas de salud, es una cosa, sistemas de servicio de atención médica es otra cosa, mucho más simple... mucho más simple.

Hablemos de esta parte que es mucho más simple nada más. Porque al final, como usted verá, la preocupación fundamental de los temas de la reforma va más a este tema...

Pero están equivocados, lean eso...

Pero entonces, ¿cómo plantea la reforma?. Nos referimos a esta última reforma que se dio en la época de la ministra Midori de Habich...

Para mí no es reforma. La única experiencia, la única oportunidad de hacer una reforma, era a través del Instituto de Gestión de Servicios de Salud - IGES, cuando salieron los decretos legislativos para la reforma de salud, se dieron las facultades, a fines del 2013. Entonces yo leí los dispositivos y me pareció una barbaridad separar del ministerio, como un pliego presupuestario independiente y adscrito al ministerio, este Instituto de Gestión de Servicios de Salud - IGES, ime pareció una barbaridad!.

\section{¿Separarlo del Ministerio de Salud?}

Pero pensándolo mejor, dije, acá puede ser la oportunidad. ¿Usted ha ido al Ministerio de Salud frecuentemente?, ¿ha recorrido los pisos?.
Si voy... las escaleras de mármol están ya...

Pero, ¿ha visto la cantidad de gente que está trabajando ahí?... Y hay más gente también trabajando en otros edificios. Hay un edificio en la avenida Arequipa.

\section{Otro está por el Parque de las Leyendas}

Hay un montón... hay tanta gente que no sabe qué hacer. Muchos que han sido mis alumnos me dicen: Doctor, no tenemos nada que hacer. Entonces, yo no me enojo... Y digo, preferible es que no hagan nada a que hagan cosas equivocadas... porque son simplemente gente que sigue, y cuando tienen otra inquietud, tienen la hostilización de los jefes.

Entonces, una de las observaciones suyas es a este proceso de reforma, como lo menciona el Ministerio de Salud, es el hecho de haber separado a este instituto, ¿Qué otras cosas?

No, pero fue una buena oportunidad y la persona que fue nombrada, era el ex ministro Oscar Ugarte; con él había tenido la oportunidad de conversar y yo le dije: "Esto es una barbaridad", y después le dije: "es una barbaridad, pero que se puede aprovechar". Porque yo no creo que el Ministerio...un mamut pesado... dormido....haga una reforma. ¡No creo que la haga!. Conozco el ministerio, pero una cosa separada, nueva, sí lo puede hacer. Después vino el nombramiento de él, y me dijo "David, me han nombrado en este cargo, jefe institucional y tú has dicho que te parecía una barbaridad pero que puede ser muy bien aprovechada para hacer una verdadera reforma, ¿por qué no me vienes a ayudar como asesor?. No te puedo pagar más de $\mathrm{S} /$. 15,000 soles, porque así está establecido los niveles". Entonces le dije, no, en esas condiciones no... S/. 15,000 soles para mi es nada, yo tengo otra ambición. Y me dijo, pero no puedo, no puedo.... S/. 15,000 soles y te puedo conseguir algunas cositas, se puede redondear entre S/. 18,000 y S/20,000 soles. No, le dije, ¡de ninguna manera!. Entonces, ¿cuánto quieres?... Esto... acepto y me rompo el alma contigo con ésta condición... aquí lo tengo en la tarjeta ¿que dice debajo de mi nombre?

\section{Ad honorem, ¿porque?}

Porque entonces te puedo decir las verdades de cuando estés equivocado. Porque un asesor no es aquel que le dice: sí señor, sí señor... a todo, si no, es el que le abre los ojos y lo critica... y te puedo criticar. Todavía conservo la tarjeta y ahí estuve hasta que lo sacaron. Y cuando lo sacaron a él, yo también renuncie. Y conozco de los entretelones, de las cosas que normalmente no se ven porque están debajo de la alfombra, me las sé de memoria todas.

Pero, ¿cómo darle coherencia a todos esos intereses políticos, para construir ese consenso que usted dice y vamos con un objetivo común?. ¿Qué es lo que predomina en las decisiones de las políticas de salud?

El problema de la educación, el problema de demostrar ejemplos. Acá lo ve usted, les propongo ver una verdadera reforma de salud, en el terreno, hecha en el terreno, sin apoyo sin hostilizamiento del Ministerio de Salud, donde van a ver todas las ideas de una verdadera reforma de salud... salud bien entendida. Vamos unos tres días a Huancavelica, a la Provincia de Churcampa, donde allí Médicos Mundi-Navarra, que es una ONG del gobierno de España-Navarra estuvo apoyando y sigue apoyando esta experiencia y quien es el representante de Médicos Mundi Navarra en el Perú es el ex ministro Fernando Carbone.

Lo conozco, también estuve en la presentación y también lo escuché a usted. ¿Qué cree que es lo que funciona ahí?

Funciona el hecho de que hay en primer lugar, participación ciudadana de verdad. En segundo lugar, apoyo, conocimiento y convencimiento para apoyar permanentemente, del gobernador regional, del alcalde provincial y de los alcaldes distritales.

¿Qué pasará cuando cambie el presidente regional?. ¿Quién defenderá eso?. Porque supongamos que cuando cambie el presidente, ¿quien puede defender que eso no cambie?

Es muy difícil... han cambiado presidentes, pero ya está todo armado y la base es los sectores. La gente que está 
ahí. Ejemplo, médicos graduados que van a hacer la residencia, llegaron ahí y quien ha dirigido todo este proceso de Churcampa, que para mí es un modelo extraordinario, no fue un médico, fue una enfermera. Entonces llegaron ahí para hacer la residencia y dijeron ino puede ser que....!. Ahí lo agarraron los demás y les convencieron a que trabajen. Hoy día son defensores de quien debe dirigir, es quien tiene la mejor capacidad.

Entonces, la presencia de la ciudadanía, los movimientos sociales, el conocimiento, le da la sostenibilidad...

¡Absolutamente!. Tienen todo, no se imagina. Tienen todas las casas, es decir para cada casa tiene una numeración, saben cuántos viven en la casa; y si nace alguien lo incorporan; si se muere alguien, lo descuentan. Es decir, mejor que cualquier explicación, es mejor ir a ver en el terreno, funciona, !funciona!.

¿Y que pasaría cuando ya terminen los recursos del proyecto de Médicos Mundi - Navarra?. ¿Se debilitará la propuesta?

No, porque hay responsabilidades. Hay convenios firmados con el gobierno regional, con la municipalidad y con el propio ministerio se ha logrado.

¿Por qué ahí sí funciona la descentralización y no como proceso general?. Un sistema así, que está funcionando en Huancavelica, que me parece que es un buen ejemplo, ¿qué posibilidades habría de manejarlo a escala nacional?, ¿cómo transferir esa experiencia a nivel nacional?

Mostrándolo, haciendo seminarios, etc. ¿Pero qué ha pasado?, hubo gente del ministerio que auspicio esto también.... los han castigado, mejor dicho las han castigado... porque estaban haciendo cosas que no correspondían a las directivas del ministerio. El sentido burocrático, lo que dice el ministerio, aunque esté mal, hay que cumplirlo.

O sea, una normativa salida desde el escritorio de un ministerio, ¿es más importante que procesos sociales, que consolidan las cosas?

¡Absolutamente!, jabsolutamente!. No hay que caer en el ejemplo del ministerio, porque lo del ministerio lo puede hacer cualquier institución, es decir, Médicos Mundi pudo haber hecho eso.

Pero yo miro desde otra arista, ¿cómo mejorar o fortalecer la capacidad rectora del ministerio?. Porque se necesita de alguien que conduzca este proceso, porque si el ministerio está muy burocratizado, ¿cómo lo mejoramos?

¿Qué diferencia hay entre liderazgo y autoridad formal?. Yo le voy a mandar un artículo que tengo sobre eso. Es decir, la autoridad formal ordena, manda, regula, obliga, nadie lo sigue. Al liderazgo lo siguen, porque le reconocen, no manda, no ordena.

Entonces, ¿podríamos decir que hay un débil liderazgo por parte del Ministerio de Salud?

¡Absolutamente!

$Y$ cómo lograr a nivel nacional también, la presencia de la participación social, de los movimientos sociales, en la construcción de políticas, o en hacer que el ministerio ejerza un liderazgo, construya un liderazgo. ¿Cómo modificar ese ministerio de salud?, porque igual, se le necesita para conducir el proceso

¡Pero claro!... depende de las personas. Las instituciones no son ni los edificios, ni los equipos, ni la tecnología, ni nada. Las instituciones son las personas, las realidades sociales son personas, si las personas son acomplejadas con complejos de inferioridad y quieren ejercer su mando, porque es mando, entonces... están perdidas. Esa es la diferencia fundamental entre liderazgo y autoridad formal.

Viendo otra arista, ¿no será también que la clase política que dirige el gobierno en un momento determinado, un presidente, no ve la importancia al tema de salud, como un proceso de ayuda al desarrollo, y de lucha contra la pobreza, contra la inequidad y no le dan prioridad?. En ese sentido, el Ministro de Salud tiene una cancha muy limitada para su maniobra

No lo creo. Lo que pasa es que a veces se nombra ministro de salud a un técnico puro. Y un técnico puro es como si usted quisiera hacer un prostíbulo con vírgenes, o con monjas. No funciona.

Es decir, que tiene que haber alguien que tenga la capacidad de ...

La capacidad de ver, de pensar, de arriesgarse, de soñar. Eso es clave... si no... no funciona. Por ejemplo, volviendo a Churucampa, que yo insisto que vayamos a verlo, porque una cosa es lo que le cuente y otra cosa es verlo. Practican el parto vertical, que es más natural. El parto horizontal al que estamos acostumbrados, es para comodidad del médico obstetra, no es para facilitar el parto de la mujer. Y el parto vertical, lo practican y yo lo he visto, incluso soy padrino de una criaturita que nació ahí, en esos días que yo estuve. Es una silla, no es completamente vertical, la mujer está sentada en un banquito y en la silla está el marido. El marido la está abrazando, le aprieta el abdomen y la ayuda, es el sentido... acá está el padre... que le dice yo te protejo y todo eso.... Y sale la criatura suavemente. $Y$ las madres, como tienen el control de todas las casas, de toditas las casas, ninguna se escapa, ya saben qué alimentación debe de tener la madre y un mes antes, la madre con el padre tienen que trasladarse.... Adaptaron en el centro de salud una casa que era para la madre y el padre con dos camitas (alojamiento).

Funciona muy bien en el tema de prevención y atención primaria. ¿Cómo se enlaza, por ejemplo si una persona allí, necesita de un trasplante, tratarse de cáncer, o de una enfermedad crónica, cómo se hace?

Ahí viene el problema de la referencia.

¿A dónde refiere Huancavelica?. ¿Los pacientes son referidos a otro nivel?

Por eso me interesaba tanto el IGES. Cuando yo me enteré que el IGES tendría que tener el control del os institutos nacionales especializados y de los hospitales nacionales más importantes, yo dije que esta era la oportunidad. Y efectivamente, han habido casos reales en el cual se ha podido traer a la persona hacia el instituto, hacia lo mejor de lo mejor que puede haber en Lima...

Ahora, y ¿el gasto o el costo de esa prestación quien la asume?

El Seguro Integral de Salud 


\section{$Y$, ¿está cubriendo todo en el país?}

Sí, sí..

\section{¿Usted cree que en ese sentido ha mejorado?}

Lo que pasa es que muchas gente todavía no va. Como están acostumbrados a que el hospital es para morir... Yo lo he visto en África. Menos mal que yo he viajado por todo el mundo. En África, yo he visto que la gente va por la vereda, y de repente cruza la calle, termina esa cuadra y regresa para seguir por este lado de la vereda y yo les pregunto ¿porque?, porque ahí hay un hospital y el hospital está asociado con muerte... Llegan al hospital muy tarde a morirse, entonces asocian hospital- muerte, y no quieren pasar cerca... eso lo he visto en África... Venga un momentito...(me conduce al interior del departamento y me conversa sobre la madre Teresa de Calcuta). Ella me dijo, sí pues, no he podido alcanzarte, tú me estás ganando 4 a 3, entonces cuando yo estoy acá en el ministerio y movilicé para que el Estado no gastara ningún centavo, sino contribuciones voluntarias de distintas instituciones, la invitamos al Perú.

Y vino la Madre Teresa... ¿Qué dice la dedicatoria?

Alma Madre, está en inglés. Ahora ya puedo pecar porque tranquilamente tengo una santa en el cielo que me va a perdonar, lo malo es que solamente puedo pecar de pensamiento... se peca de obra, de omisión, de palabra y de pensamiento... y solo me queda pecar de pensamiento, pero entonces de pensamiento los pecados pueden ser terribles. Pase por acá... (Me enseña un mapa mundial con alfileres de varios colores), estos son todos los países donde he estado en función de trabajo. Este es un regalo de mi secretaria de Ginebra. Mi obligación era estar por lo menos dos veces al año en las seis oficinas regionales de la OMS.

Las estrellas rojas, ¿que significan?

Actividades docentes.

\section{¿Las azules?}

Proyectos nacionales y las verdes proyectos específicos.

¡Qué bien!. Lo felicito doctor, una gran obra. Para usted, ¿cual le parece uno de los mejores sistema de salud, de todos los que usted conoce?

El sistema de salud en Israel. Es un seguro único, se llama ISTRA DUT, es único, no hay contribución, es del Estado, para todos, de impuestos. Y los impuestos son cargados a los productos que producen enfermedades. Usted va de visita, desciende del avión en Tel Aviv, en Jerusalén, en donde sea y se resbala y se cae, usted no es israelí, usted es extranjero... lo cubre el seguro desde el momento que pisa Israel.

Que interesante. O sea que allá el gasto de bolsillo es cero.

No hay.

\section{$Y$, ¿acceso a todas las prestaciones?}

A la que le corresponda de acuerdo a lo que necesite.

\section{¿Cuál es la gran diferencia con Perú?}

Acá solamente tiene la clase privilegiada que tiene seguros privados, el Seguro Social, que está muy mal llevado a mi modo de ver y ahora un financiamiento SIS, porque el Seguro Integral de Salud, simplemente es un financiamiento, pero que está ayudando mucho...

¿Mucho?. ¿A pesar de que el per cápita es muy bajo que no alcanza para todas las prestaciones?

No alcanza para todas las prestaciones, pero sí cubre.

\section{¿Qué me iba a enseñar acá?}

Estos son todos los lugares de mi experiencia de vida directa. Por ejemplo, yo tenía que estar por lo menos dos veces al año, y eso fue durante trece años, en las oficinas regionales. Para las Américas, la oficina regional está en Washington; para Europa la oficina regional está en Copenhague, Dinamarca; para el Mediterráneo la oficina principal está en Alejandría, Egipto. ¿Y porque Alejandría?, porque Alejandría fue la primera sede de la Organización Mundial de la Salud que se hizo después de la primera guerra mundial y que después no tuvo continuidad. Para África está en Trazamil, Congo; para el sud este de Asia está en Nueva Delhi, India, y para el pacifico occidental está en Manila, Filipinas.

\section{¿Usted vive aquí solo en este depar- tamento?}

Sí, solo. Estamos en uno de los lugares céntricos de la ciudad, Miraflores. Y acá tiene el último reconocimiento que me dieron.
Dice: "Héroe de la Salud Publica de Ias Américas, 2012 - Dr. David Tejada De Rivero, en reconocimiento a su sobresaliente contribución a mejorar la salud de los pueblos de las américas y del mundo - Organización Panamericana de la Salud, Lima, Marzo 2012". iLo felicito doctor!

Cuando yo recibo esto en una ceremonia, ahí en la OPS y vino desde Ginebra la directora de la OMS, y cuando estaba en la ceremonia dije que yo tenía muy merecido ese premio. ¿Por qué?, casado tres veces, divorciado tres veces... requiere heroísmo ¿̇o no?

Siempre ha sido muy reconocido su muy buen sentido del humor doctor

Claro, en la vida las cosas hay que tomarlas así.

Una de las cosas que quisiera escuchar de usted es su opinión sobre la descentralización....

¡La descentralización muy mal llevada a cabo!. La descentralización ha dificultado todo. En este momento por ejemplo, hay que reconocer, el Ministerio de Salud no tiene ya ninguna injerencia en las regiones. ¿Por qué?, porque las regiones han tenido no solamente transferencia de fondos, que no saben qué hacer y no lo pueden gastar. No solamente en salud, si no en todo en general, si no que han tenido la transferencia en el sentido de que les han dado todas las facilidades para que ellos se conviertan en lo que yo \|lamo "no es descentralización... es neofeudalizacion". Cada gobierno regional es un feudo, con un señor feudal; y entonces, el que era antes director regional de salud, designado por el Ministerio de Salud, ahora ya no se llaman directores, le cambiaron el nombre y son designados a voluntad del presidente regional (que ahora de común acuerdo ya no se llama presidente regional, sino gobernador regional), sin tener en cuenta ningún requisito ni nada: "gente de confianza".

No hay procesos de selección, ni de méritos...

Sin méritos... nada de eso. Entonces, esa persona para seguir teniendo la confianza del presidente regional, hacen como los coroneles que decían: "mi general, defenderemos la independencia", y entonces no quieren saber nada del 
ministerio, para poder ser fieles al presidente regional.

\section{¿Cómo mejoramos eso?}

Cambiar la legislación completa.

¿Para que nuevamente se centralicen algunas actividades?

No. Que se descentralice, pero todo regulado.

Entonces, ¿solo sería una desconcentración de ciertas funciones o persistir por la descentralización pero más regulada?

Persistir con la descentralización pero bien regulada; y que el Ministerio de Salud cumpla su función rectora. La función rectora es que se reconoce que el ministerio tiene la razón, esa es la función rectora, ese es el liderazgo.

Viendo para atrás, cuando usted fue ministro...

Yo fui ministro en 1985 y 1987. La primera vez fue casi dos años (falto una semana para que sean dos años) y la segunda vez fue apenas cinco meses.

En el Perú, ¿cuánto se ha avanzado en temas del sistema de salud?

En primer lugar, yo le diría, lamento mucho tener que decirle: sistema de salud no existe. Lo que existe es sistema de atención médica reparativa, es lo que existe acá en el Perú y seguramente en todas partes.

Ese sistema de atención médica reparativa, ¿se ha fortalecido?, ¿cuánto se ha avanzado?

Puede haberse fortalecido en algunos aspectos, pero no mucho porque también hay el problema del financiamiento. El problema de este sistema de atención médica es que está muy sujeto a la voracidad de la industria productora de equipos de diagnóstico complementario.

\section{$Y$ de medicamentos...}

Pero ahora están desplazando a los medicamentos, los equipos. Incluso hay artículos escritos en Estados Unidos, como critican el hecho de que los equipos que están produciendo, en lugar de tener una duración de diez años o veinte años, tienen una duración de dos años, eso como punto uno. Punto dos, no hay repuestos, no producen repuestos.

\section{¿Son equipos de una sola vida?}

De una sola vida de dos años, reducida. Porque lo que les interesa a ellos no es la salud de la gente. ¡Lo que les interesa a ellos es ganar!, iganar dinero!. Y nosotros todos idiotas estamos pendientes... y el médico idiota, malformado, piensa que solamente se realiza profesionalmente si trabaja con lo último de la tecnología. Pero no se pone a pensar en el costo social, el costo humano que significa ese latrocinio organizado. Hillary Clinton, cuando fue esposa del presidente Clinton, ella muy inteligente...

Bueno, todavía sigue siendo esposa... cuando Clinton era el presidente.

Si, cuando el marido era el presidente, ahora va a ser posiblemente candidata presidencial. A mi ella me gusta mucho, sobre todo porque ella en esa época buscó los mejores técnicos para el estudio sobre la atención médica... no salud... medical care; hizo un estudio y llegaron a la conclusión de que la economía en Estados Unidos en determinado momento sería absolutamente incapaz de absolver los gastos crecientes de la tecnología...

Lo que está pasando en Estados Unidos, también en Canadá...

Ese fue el Informe Clinton. Ese informe Clinton la esposa del Presidente que lo hizo con los mejores técnicos, lo presentó al Senado, y el Senado nunca lo vio. Cuando Hillary Clinton es nombrada por Obama Secretaria de Estado, ella reconstituye el equipo con nueva gente, reactualiza el informe y como Secretaria de Estado va y lo presenta al Senado. El Senado nunca lo vio y ha aparecido un trabajo de investigación en Estados Unidos, donde dice ¿por qué no se ha visto en el Senado dos veces?.

\section{¿Cuál es la razón?}

La razón es, comprobado, la mayoría de los senadores republicanos y la mayoría de los senadores demócratas, fueron financiadas sus candidaturas al senado por la industria.

Para el futuro, ¿qué prácticas de alta gestión, de políticas públicas, usted cree que son necesarias?. ¿Qué recomendaría en este tema de seguir fortaleciendo el sistema de salud?

Yo creo que es clave la formación del personal, la preparación a todos los niveles. Creo que fue un gran error, hecho ex profesamente, por Fujimori, de cerrar la Escuela de Salud Publica.

$Y$ que ya se está empezando nuevamente a retomarla..

Espero. Yo siempre he estado reclamando la Escuela de Salud Pública. Yo fui invitado para que vean como es la cosa, con un grupo llamado de expertos y tengo la relación. Me gustaría tener las hojas de ruta para estos expertos porque no los conozco. He preguntado y tampoco los conocen.... bueno... estuvo conmigo también como invitado Oscar Ugarte, entonces nos presentan todas las ideas que tienen ellos, no nos dan ningún documento de anticipación técnica, termina la presentación y dicen: bueno, esta es la primera reunión, ya convocaremos a la segunda reunión dentro de dos semanas; entonces yo pido la palabra y digo: por favor dentro de dos semanas yo quiero tener los documentos que han presentado ustedes en power point antes, para poder estudiarlos.... Ahora, no nos han dejado ni intervenir, ni opinar, nada, no puede ser, yo quisiera que por favor, por lo menos con una semana de anticipación, yo y todos nosotros recibamos la información para poder estudiarla y venir a colaborar. Hasta el día de hoy no hemos sido citados y no hemos recibido nada. ¿Qué significa eso?.

Es justo lo que se critica, que en términos de construir una política pública, en términos de construir procesos consolidados se necesita la participación de otros, el consenso...

Es indudable. Es indudable.

Doctor, le quiero agradecer mucho por sus opiniones, por su información, por habernos trasladado esta experiencia suya. ¿Cuál es su edad?

50, ino miento!. Cincuenta con s...

$Y$ no entendí eso de tres matrimonios y cuatro divorcios

No, tres matrimonios y tres divorcios. 
Pero mantengo muy buenas relaciones con mis ex esposas, me comunico con ellas vía Skype, internet, etc. Porque tenemos hijos, tres, más dos, más dos, son siete hijos.

\section{Muchas gracias doctor}

Bueno, espero que haya contribuido con algo. Ahora, lo importante sería ver ¿cómo resolver el problema?. Tratar de hacer salud... no esperar a que la gente se enferme para curarla bien.

\section{Es un tema intersectorial...}

¡Absolutamente intersectorial!. Y para mí la rectoría seria cuando el ministerio sea capaz de que todos los demás ministerios, toditos sin excepción y todas las otras instituciones nacionales que no sean ministerios, tengan políticas de salud que formen parte de un solo programa.

Pero ahí fijese, tanto para conducir este proceso intersectorial, para construir salud, como para dirigir el sistema de atención médica, se necesita de un ministerio sólido, fuerte...

La clave es con gente capaz, icon gente que piense!. ¡No con gente que esté sentada para firmar papeles!. Y acá no- sotros ponemos la propuesta, ¿qué dice aquí?...

"Una auténtica reforma se inicia operativamente en la ciudadanía"

¡Claro!. La ciudadanía enseña, la experiencia de Churcampa... anótelo Churcampa. Asumo la palabra de Fernando Carbone, para invitarlos y podemos viajar los tres a Churcampa, estar un par de días para ver en el terreno todo esto que hablamos... ihecho en el terreno!. Y no hecho por nosotros, sino por la gente de ahí, ese es el mérito, el mérito grande.

Lima, junio de 2015 